\title{
Blackbox And Accident Prevention System in Automobiles
}

\author{
$1^{\text {st }}$ Kannan S \\ VLSI \& Embedded Systems \\ ER\&DCI Institute of Technology \\ Thiruvananthapuram, India
}

\author{
$2^{\text {nd }}$ Kadar A A \\ VLSI \& Embedded Systems \\ ER\&DCI Institute of Technology \\ Thiruvananthapuram, India
}

\author{
$3^{\text {rd }}$ Divya D S \\ VLSI \& Embedded Systems \\ ER\&DCI Institute of Technology \\ Thiruvananthapuram, India
}

\begin{abstract}
As the number of vehicles on the roads is increasing at an alarming rate, so is the number of accidents and the fatalities that result. Hence a safer means is of utmost necessity. The proposed system incorporates a Blackbox facility similar to that used in aircrafts and an accident prevention and alert system. Blackbox maintains a catalogue of data regarding the automobile that can be retrieved in the event of an accident. Prevention mechanism alerts the driver regarding possible dangers approaching that may lead to an accident. Blackbox will keep a record of the location of the vehicle, speed, temperature, seatbelts status, door status etc. The data can be stored within the box and can be retrieved and analyzed for future references. Prevention mainly uses speed and distance measurements. Alert signals are generated if distance between cars goes below the threshold set value. Also sudden deceleration is monitored.
\end{abstract}

Index Terms-Blackbox, accident prevention

\section{INTRODUCTION}

An accident is an unplanned event that sometimes has inconvenient or undesirable consequences, other times being inconsequential. The occurrence of such an event may or may not have unrecognized or unaddressed risks contributing to its cause.

A traffic collision, also called a motor vehicle collision (MVC) among other terms, occurs when a vehicle collides with another vehicle, pedestrian, animal, road debris, or other stationary obstruction, such as a tree, pole or building. Traffic collisions often result in injury, disability, death, and property damage as well as financial costs to both society and the individuals involved. Major contributing factors of accidents are

- Vehicle design

- $\quad$ Speed of operation

- Road design

- Impairment due to alcohol or drugs

- Behavior, notably distracted driving,

- $\quad$ Speeding

- $\quad$ Street racing.

Traffic collisions can be classified by general types. Types of collision include head-on, road departure, rear-end, side collisions, and rollovers.

Effects of a vehicular accidents are both physical and psycho- logical. A number of physical injuries can commonly result from the blunt force trauma caused by a collision, ranging
From bruising and contusions to catastrophic physical injury (e.g., paralysis) or death. Following collisions, longlasting psychological trauma may occur. These issues may make those who have been in a crash afraid to drive again. In some cases, the psychological trauma may affect individuals' life can cause difficulty to go to work, attend school, or perform family responsibilities. Major accidents are caused by the human factor. Distracted drivers cause majority of accidents. These distractions vary from mobile usage to children or pets. Over- speeding, miscalculations of distances, lack of on road ex- presences, impairments in hearing or vision. These along with carelessness is a mixture for accidents on the road. DUI or Driving under Influence is a punishable offence and is also a major contributor of traffic collisions. Intentional accidents also occur in which drivers intentionally causes accidents to harm others or themselves. Young unqualified drivers without licenses also cause accidents. Causes due to human factor also includes sleep deprivations during long night drives.

Conditions of roads can cause accidents. Slippery roads, wrong banking of roads can cause even experienced drivers to lose control of vehicles.

Vehicle design and maintenance also has a role in traffic collisions. Poor maintenance and lack of servicing can cause cars to backfire, brake failures, engine clogging etc. Drivers and passengers not wearing their seat belts results in more fatalities because in new automobiles the airbags will not deploy if seat belts are not closed and injuries sustained will be deadlier.

Approximately 1.35 million people die in road crashes each year, on average 3,700 people lose their lives every day on the roads. Road traffic injuries are the leading cause of death among young people aged 5-29. Young adults aged 15-44 account for more than half of all road deaths.

Hence in this era of advanced technology a system which provides a safer means of transport in the busy roads is of utmost necessity.

So in this project I intend to implement a Black-Box in the vehicles along with other accident prevention systems and an accident alert system as a worst case scenario. 


\section{LITERATURE SURVEY}

Abdallah Kassem et.al in "Vehicle Black Box System" proposes a prototype of a blackbox system for vehicles. VBBS in the proposal can contribute to constructing safer vehicles, improve treatment of crash victims and aid in search and rescue .Prototype could be designed with minimum number of circuits and comprises of speed sensors, water sensors, light sensors, accident and belt sensors.[1]

D.Guru Pandi et.al in "Embedded Based Accident Prevention Technique using Image Processing" specifies about accident prevention by monitoring the roads. Images of objects ahead are captured and processed and if user fails to detect the obstacles alerts are generated by monitoring the brake sensors

If proper response to the alert is not observed a driver circuit is used to apply the brakes .Automatic brakes compensates for lower response time of humans. [2]

Sayanee Nanda et.al in "An IOT Based Smart System for Accident Prevention and Detection" put forth a system that checks whether the driver is drowsy or in an unstable state which can lead to pedal mix-up and in some cases unintended acceleration or turning of the steering wheel to the wrong direction which can lead to crashing of the vehicle with other vehicles or concrete road barrier. [3]

\section{EXISTING SYSTEM}

\section{A. $O B D$}

On-board diagnostics (OBD) is an automotive term referring to a vehicle's self-diagnostic and reporting capability. OBD systems give the vehicle owner or repair technician access to the status of the various vehicle sub-systems. The amount of diagnostic information available via OBD has varied widely since its introduction in the early 1980s versions of on- board vehicle computers. Early versions of OBD would simply illuminate a malfunction indicator light or"idiot light" if a problem was detected but would not provide any information as to the nature of the problem. Modern OBD implementations use a standardized digital communications port to providereal- time data in addition to a standardized series of diagnostic trouble codes, or DTCs, which allow a person to rapidly identify and remedy malfunctions within the vehicle.

\section{B. Data Loggers}

Data loggers are designed to capture vehicle data while the vehicle is in normal operation, for later analysis. Data logging uses include: Engine and vehicle monitoring under normal operation, for the purpose of diagnosis or tuning. Some US auto insurance companies offer reduced premiums if OBD- II vehicle data loggers or cameras are installed - and if the driver's behavior meets requirements. This is a form of auto insurance risk selection.

\section{SYSTEM REQUIREMENTS}

\section{A. ARDUINO MEGA 2560}

The Arduino Mega 2560 an ATmega2560 based microcontroller. It has 54 digital input/output pins, 16 analog inputs,
4 UARTs, a $16 \mathrm{MHz}$ quartz oscillator, a USB connection, a power jack, a reset button, and an ICSP header. It has got everything needed to support the microcontroller. Simply connect it to a computer using a USB cable or power it with an AC-to-DC adapter or battery to get started. The Mega 2560 board is often programmed with the Arduino Software (IDE).The ATmega2560 has got $256 \mathrm{~KB}$ of flash memory for code storage, $8 \mathrm{~KB}$ of SRAM and $4 \mathrm{~KB}$ of EEPROM. Each of the 54 digital pins on the Mega can be used as an input or output, using the functions: pinMode(),digitalWrite(), and digitalRead(). They operate at 5 volts.

\section{B. GSM MODULE}

The SIM900A is a complete Dual-band GSM/GPRS solution, which may be embedded within the customer applications. It delivers GSM/GPRS 900/1800MHz performance, as per industrial standards, for voice, SMS, data, and fax during a small form factor and with low power consumption. SIM900A can be used in most space requirements in user applications, especially for slender and compact demand of design.

\section{GPS MODULE}

The NEO-6M GPS module is a well-performing complete GPS receiver with a built-in $25 \times 25 \times 4 \mathrm{~mm}$ ceramic antenna, which provides a strong satellite search capability. With the power and signal indicators, you can monitor the status of the module.

\section{ULTRASONIC SENSOR}

The HC-SR04 ultrasonic sensor uses sonar to determine distance to an object like bats or dolphins do. It offers excellent non-contact range detection with high accuracy and stable readings in an easy-to-use package. From $2 \mathrm{~cm}$ to $400 \mathrm{~cm}$ or 1 " to 13 feet.

\section{E. SPEED SENSOR}

IR speed sensor module with the comparator LM393.If anything is passed between the sensor slots, it creates a digital pulse on the D0 pin. This pulse goes from $0 \mathrm{~V}$ to $5 \mathrm{~V}$ and is a digital TTL signal.

\section{F. TEMPERATURE SENSOR}

LM35 is an integrated analog temperature sensor whose electrical output is proportional to Degree Centigrade. LM35 Sensor does not require any external calibration or trimming to provide typical accuracies.

\section{G. IR TRANSMITTER \& RECEIVER}

IR Transmitter and receiver are used to control any device wirelessly, means remotely. TV remote and TV are the best example of IR transmitter and receiver. TV generally consist TSOP1738 as the IR receiver, which senses modulated IR pulses and convert them into electrical signal.

\section{H. SD CARD MODULE}

An SD Card Module or a Micro SD Card Adapter is asimple board which facilitates connection between a Micro SD card and a Microcontroller like Arduino. 


\section{DC MOTOR \& DRIVER}

The DC motor drive is a type of amplifier or power modulator that integrate between the controller and a DC motor. It takes the low current and then converts it into a high current which is appropriate for the motor.

\section{J. PUSH BUTTON}

A push button switch is a small, sealed mechanism that completes an electric circuit when you press on it. When it's on, a small metal spring inside makes contact with two wires, allowing electricity to flow. When it's off, the spring retracts, contact is interrupted, and current won't flow.

\section{PROPOSED SYSTEM}

Blackbox will contain a SD card module from which the SD card can be retrieved and will contain all necessary information. Data stored are

- $\quad$ Speed using speed sensor

- Temperature using temperature sensor

- $\quad$ Location using GPS modules

- $\quad$ Seat belt statuses using push buttons

The prevention system monitors the distance from following cars using ultrasonic sensors and sends an alert signal to the driver if the threshold distance is crossed.

Speed sensors are used for monitoring over speeding. Incase of over speeding initially alerts are given and if proper response is not observed speed is reduced by the driver circuit. Also sudden deceleration of the car is monitored using same speed sensors and an alert is passed to car the immediately following so as to avoid a rear end collision. The alert can be sent using an IR transmitter module and a receiver module in the car behind.

Speed sensors will be attached near the wheels of the car and hence a motor is used to model that. An encoder disc is used for the optocoupler sensor.

A GSM module is also incorporated to send SOS alert in case of an accident. Push buttons are used to model accidents.

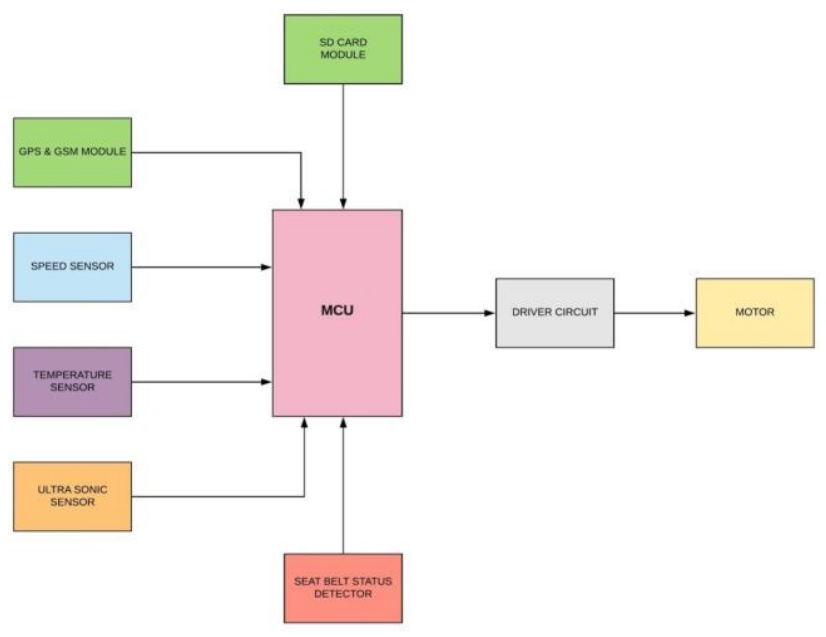

Fig. 1. Block Diagram.

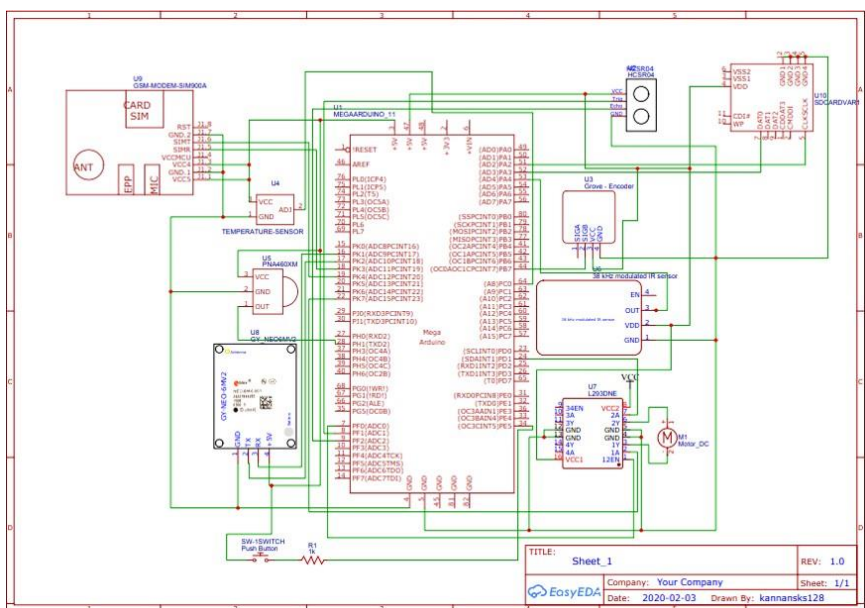

Fig. 2. Circuit Diagram

The MCU or Microcontroller unit used is the Atmega 2560 in the Arduino Mega developer board. The speed of the motor is controlled using a potentiometer (pot).Over speeding and sudden braking is achieved by changing the resistance of the pot. Simple two legged push buttons are used for seatbelts and accidents modelling. If pressed one push button is programmed to indicate seatbelt closed and the other, an accident has occurred and SOS message is sent. If the accident push button is pressed then data pushing to SD card is stopped so that the data corresponding to the time of accident is obtained by retrieving the SD card.

\section{EXPERIMENTAL RESULTS}

The data from LM35 temperature sensor, optocoupler speed sensor, seatbelt status via push button, rear end distance from ultrasonic sensor and coordinates(latitude \& longitude) from Neo 6M GPS module is stored in the SD Card as real time data. When the speed crosses a preset safe threshold value an over speed alert is generated. When the vehicle is decelerating at a rapid rate than usual an alert signal is sent to the vehicle immediately following via IR signal generator. When the vehicle immediately following crosses apreset safe threshold distance, the driver is alerted. As a worst case scenario, in the unfortunate event of an accident a SOS message is sent to the stored numbers.

\section{CONCLUSION AND FUTURE ENHANCEMENTS}

The proposed system will help in developing a safer means of transportation as it helps in prevention of accidents and also in the event of occurrence of an accident alerts are sent so as to increase the help and rescue of the victims. The overall system is cost effective and efficient in all aspects. The proposed system will aid in search and rescue and helps in post-accident investigations.

Future enhancements could include autonomous driving systems, image processing methods for obstacle detection, manoeuvring mechanism for obstacle avoidance and other accident prevention mechanisms. 


\section{REFERENCES}

[1] Abdallah Kassem, Rabih Jabr, Ghaby Salamouni."Vehicle Black Box System" IEEE International Conference, Montreal Canada. April 7- 10, 2008.

[2] D.Guru Pandi, J.Navarajan, R Vishal.”Embedded Based Accident Pre- vention Technique using Image Processing" IJARIIE-ISSN(O) 2395- 4496,Vol-2,Issue-2,2016.. (VLSI) Syst., vol. 17, no. 8, pp. 1127-1137,Aug. 2009

[3] Sayanee Nanda, Harshada Joshi, Smita Khairnar." An IOT Based Smart System for Accident Prevention and Detection" 2018 Fourth International Conference on Computing Communication Control and Automation, IEEE, 25 April 2019 